\title{
On the Computation of Nonhyperbolic Fixed Points
}

\author{
Mário M. Graça
}

\section{CONTENTS}

1. Introduction

2. Combined and Flat Iteration Functions

3. Applications

Acknowledgements

References

2000 AMS Subject Classification: Primary 47H99, 65H05; Secondary 32S05, 58K05

Keywords: Fixed point, nonhyperbolic, super-attracting fixed point, iteration function, order of covergence
In order to deal with nonhyperbolic fixed points of a given real iteration function $g$, we construct new iteration functions $C$ which will be called combined. When a nonhyperbolic fixed point of $g$ becomes a super attractor fixed point of $C$, the iteration function $C$ is called flat.

Some flat iteration functions are constructed based on Newton's iteration function. Several numerical examples illustrating the good properties of flat iteration functions are presented.

\section{INTRODUCTION}

A nonhyperbolic (or neutral) fixed point $x^{*}$ of a real iteration function $g$ is a point satisfying $\left|g^{\prime}\left(x^{*}\right)\right|=1$. The numerical computation of a nonhyperbolic fixed point $x^{*}$ of a given map $g$ using the iterative process $x_{n}=g\left(x_{n-1}\right)$ is, in general, useless in the sense that it will involve a large number of iterates with a corresponding growth of computational errors.

The problem of computing nonhyperbolic fixed points $x^{*}$ such that $g^{\prime}\left(x^{*}\right)=1$ is equivalent to the computation of the solution of the equation $f(x)=0$ where $f(x)=x-g(x)$ and $f^{\prime}\left(x^{*}\right)=0$. The mathematical study of this sort of problem is called singularity theory. Although the case studied in this work refers only to real maps, the underlying problem for higher-dimensional maps is the computation of the solution of a set of nonlinear equations $f(x)=0$ where $x, f(x)$ are in $\mathbb{R}^{n}$ and the Jacobian matrix of $f$ does not have full rank $n$. The classical reference for singularity theory is [Golubitsky and Schaeffer 85] and for its numerical implementation, see [Govaerts 00], Chapters 6 and 7.

The main aim of this work is to give a general procedure for the construction of iterative processes able to compute a nonhyperbolic fixed point of a real iteration function $g$ in few steps and with high precision. A new iteration function, $C=C(g, h)$, is constructed by making a suitable combination of $g$ with another function $h$. The iteration function $C$ will be called combined.

(c) A K Peters, Ltd 1058-6458/2001 \$0.50 per page Experimental Mathematics 11:3, page 477 
We will give particular attention to those combined iteration functions (IF) constructed from an initial iterative process convergent to a nonhyperbolic fixed point, able to generate iterative processes with order of convergence of at least 2 . These combined iteration functions will be called flat (at $x^{*}$ ).

Any combined iteration function $C(g, h)$ has the property of transforming a nonhyperbolic (NH) fixed point $x^{*}$ for $g$ satisfying $g^{\prime}\left(x^{*}\right)=1$ into a super-attracting fixed point for $C$, that is $C^{\prime}\left(x^{*}\right)=0$. In other words, the iterative process associated with $C$ will have faster convergence than the process associated with $g$.

The main underlying idea is to use some iterates of a computationally inefficient iterative process to construct another one giving the nonhyperbolic fixed point in a few iterations. For instance, the iterative process $x_{n+1}=$ $N\left(x_{n}\right)$ associated with the Newton map $N(x)=x-$ $f(x) / f^{\prime}(x)$ for $f(x)=x-g(x)$ is not appropriate for the computation of a nonhyperbolic fixed point of $g$ since this point is a singularity of $f$. However, as we shall show in Section 3.1, the combined function of $g$ with $N$ is a map for which $x^{*}$ is a super-attracting fixed point.

Although in the present work only real maps are considered, the definition of our combined iteration function can be easily adapted to higher-dimensional maps. In a forthcoming paper, we will generalize the results to higher dimensions where the applications become more interesting.

This paper is organized as follows. In Section 2., we define a combined IF, $C$, and show that whenever the nonhyperbolic fixed point $x^{*}$ of $g$ is such that $g^{\prime}\left(x^{*}\right)=1$, then $C(g, h)$ is always flat (Theorem 2.5). In Theorem 2.7 , we give explicitly a flat iteration function $C(g, h)$, for $g$ satisfying some mild hypotheses of differentiability and having either a hyperbolic or nonhyperbolic fixed point.

In Section 3., we study the flat iteration functions $N_{C}$ and $N_{H}$ based on Newton's method for root finding, and we give some numerical examples illustrating the computational efficiency of our combined iteration functions. The main result of this section (Theorem 3.2) states that either for simple or multiple roots of $f(x)=0$, the iterative processes generated by $N_{C}$ and $N_{H}$ have order at least 2 , if they are convergent.

Some numerical examples are given to illustrate the main properties of our combined IF compared to the given initial IF. In particular, in Examples 1 and 3, we chose two iteration functions which attracted the attention of many authors due to the difficulties of getting good approximations for the respective nonhyperbolic fixed points. It is shown that combined iteration func- tions produce highly precise approximations in three or four iterations.

Newton-like iterators have recently received renewed attention ([Gilbert 94, Gerlach 94, Epureanu and Greenside 98]) and their comparison with $N_{C}$ and $N_{H}$ will be the subject of a forthcoming paper.

\section{COMBINED AND FLAT ITERATION FUNCTIONS}

An iterative process with iteration function $g, x_{n+1}=$ $g\left(x_{n}\right)$, if convergent to a nonhyperbolic fixed point of $g$, converges very slowly. We construct a new iteration function $C$ based on $g$, such that a nonhyperbolic fixed point of $g$ becomes an attractor point for $C$.

Definition 2.1. (Combined iteration function.) Let $g$ and $h$ be two differentiable functions in a neighborhood $D$ of a common fixed point $x^{*}$. A combined iteration function $C(g, h)$ is defined by

$$
\begin{aligned}
C(g, h)(x) & =C(x)=\frac{h(x)-g(x) h^{\prime}(x)}{1-h^{\prime}(x)} \text { and } \\
h^{\prime}(x) & \neq 1, \quad \forall x \in D .
\end{aligned}
$$

Note that $C$ is well-defined on nonhyperbolic fixed points of $g$, although it is not always defined at nonhyperbolic fixed points of $h$. This, however, is not restrictive since for a given iteration function $g$, one can choose an $h$ with $h^{\prime}\left(x^{*}\right) \neq 1$.

The requirement that $x^{*}$ be a common fixed point of $g$ and $h$ in the definition of $C$ implies that $x^{*}$ is also a fixed point of $C$. Unfortunately, the converse is not true as can be seen in Example 3.6 of Section 3. where $C$ has fixed points that are not fixed points for $g$. In the literature, these new fixed points are called extraneous (see [Vrscay and Gilbert 88]).

A hyperbolic fixed point $x^{*}$ of a map $g$ is called an attractor if $0<\left|g^{\prime}\left(x^{*}\right)\right|<1$, a repeller if $\left|g^{\prime}\left(x^{*}\right)\right|>1$, and a super attractor if $g^{\prime}\left(x^{*}\right)=0$ (see [Holmgren 96]).

Proposition 2.2. A common fixed point $x^{*}$ of $g$ and $h$ is a super-attracting fixed point of $C(g, h)$ whenever $g^{\prime}\left(x^{*}\right)=$ 1 or $h^{\prime}\left(x^{*}\right)=0$.

Proof: As $C^{\prime}\left(x^{*}\right)=\frac{h^{\prime}\left(x^{*}\right)}{1-h^{\prime}\left(x^{*}\right)}\left(1-g^{\prime}\left(x^{*}\right)\right)$, the result follows.

Proposition 2.2 gives a good property of the combined IF, $C$, showing that the nonhyperbolic fixed points of 
$g$ satisfying $g^{\prime}\left(x^{*}\right)=1$ become super-attracting fixed points of $C$. Notice that if $h$ is chosen such that $x^{*}$ is a super attractor, then $x^{*}$ is always a super-attracting fixed point for $C(g, h)$ whenever $x^{*}$ is either a hyperbolic or nonhyperbolic fixed point of $g$.

It is worth noting that the super-attractivity of the fixed point $x^{*}$ of $C$ given in the last proposition could also be obtained when $g^{\prime}\left(x^{*}\right)=-1$ if one had adopted the following definition of $C$ :

$$
C(g, h)=\frac{h+g h^{\prime}}{1+h^{\prime}} \text { with } h^{\prime}\left(x^{*}\right) \neq-1
$$

We now mention a particularly well-behaved combined iteration function which will be used throughout this work. Let $C(x, g)$ be the combination of the identity function with an iteration function $g$ having a hyperbolic fixed point $x^{*}\left(\left|g^{\prime}\left(x^{*}\right)\right| \neq 1\right)$ :

$$
C(x, g)=C(x)=\frac{g(x)-x g^{\prime}(x)}{1-g^{\prime}(x)},
$$

$\left(x^{*}\right.$ is a hyperbolic fixed point of $g$ ). has:

Applying Proposition 2.2 with $g=x$ and $h=g$, one

Corollary 2.3. Either repelling or attracting fixed points of $g$ are super-attracting fixed points of $C(x, g)$.

Notice that $C(x, g)$ is just the Newton iteration function for $f(x)=0$, with $g(x)=x-f(x)$, if $x^{*}$ is a simple root of $f(x)=0$ (see Section 3.).

Those combined functions for which the common fixed point $x^{*}$ of both $g$ and $h$ is a super-attracting fixed point of $C$ will be called flat iteration functions. The main motivation for calling these functions flat at $x^{*}$ is the following geometric argument: While the graph of $g$ in a neighborhood of $\left(x^{*}, g\left(x^{*}\right)\right)$ is like the graph of the identity function, the graph of $C$ in the same region looks like a horizontal line.

Definition 2.4. (Flat iteration function.) Let $x^{*}$ be a common fixed point of $g$ and $h$. A combined iteration function $H=C(g, h)$ is called a flat iteration function at $x^{*}$, or flat for short, if $H^{\prime}\left(x^{*}\right)=0$. If $H$ is flat and $H^{(2)}\left(x^{*}\right)=0$, we say that $H$ is a super-flat iteration function.

A flat iteration function constructed from $g$ has the property of transforming a slow convergent iterative process associated to $g$ into an iterative process associated to $H=C(g, h)$ converging faster.
Hereafter, we assume that $g$ and $h$ are sufficiently differentiable and that all the results are local, that is they are only valid in a neighbourhood of an isolated fixed point.

Theorem 2.5. For any two functions $g$ and $h$ satisfying

$$
g\left(x^{*}\right)=h\left(x^{*}\right)=x^{*}, \quad g^{\prime}\left(x^{*}\right)=1 \quad \text { and } \quad h^{\prime}\left(x^{*}\right) \neq 1,
$$

the iteration function $H=C(g, h)$ is flat. Furthermore, $H$ is super flat if and only if

$$
h^{\prime}\left(x^{*}\right) g^{(2)}\left(x^{*}\right)+h^{(2)}\left(x^{*}\right)=0 .
$$

Proof: By Proposition 2.2, $H^{\prime}\left(x^{*}\right)=0$, that is $H$ is flat and

$$
H^{\prime \prime}\left(x^{*}\right)=-\frac{h^{\prime}\left(x^{*}\right) g^{(2)}\left(x^{*}\right)+h^{(2)}\left(x^{*}\right)}{1-h^{\prime}\left(x^{*}\right)},
$$

so, the result follows.

Note that for a given iteration function $g$ with a nonhyperbolic fixed point $x^{*}$ such that $g^{\prime}\left(x^{*}\right)=1$, any other IF $h$ for which $x^{*}$ is either a repelling or attracting fixed point can be used to generate the flat iteration function $H=C(g, h)$.

The main question now is how to choose $h$ in order to obtain a flat iteration function from a given iteration function $g$, in particular when $g$ has a nonhyperbolic fixed point $x^{*}$. Although from the definition of combined iteration function, we can choose $h$ to be any function for which $x^{*}$ is a hyperbolic fixed point, from the computational viewpoint, the right choice must be one obtained from the data of the problem, that is from $g$. So, one of the first natural choices for $h$ that one can think of is the iteration function given in Equation (2-2), that is $C(x, g)$.

In fact, if $g$ has an hyperbolic fixed point $x^{*}$, then by Corollary 2.3, $h=C(x, g)$ is such that $h^{\prime}\left(x^{*}\right)=0$ and so $h$ can be used in $C(g, h)$. In the case $g^{\prime}\left(x^{*}\right)=-1$ Proposition 2.2 applied with $g=x$ gives $h^{\prime}\left(x^{*}\right)=0$. The main problem in using $C(x, g)$ as $h$ in $C(g, h)$ is when $g^{\prime}\left(x^{*}\right)=1$; in this case $C(x, g)$ is not defined.

We show that under quite general differentiability assumptions on $g$ a continuous extension of $C(x, g)$ can be used as $h$ in $C(g, h)$ in order to produce a flat iteration function.

Consider $x^{*}$ to be an isolated fixed point of $g$ such that $g^{\prime}\left(x^{*}\right)=1$ and $h$ given by

$$
h(x)=\left\{\begin{array}{lll}
\frac{g(x)-x g^{\prime}(x)}{1-g^{\prime}(x)} & \text { if } \quad x \neq x^{*} \\
x^{*} & \text { if } \quad x=x^{*} .
\end{array}\right.
$$


Let $g: D \subset \mathbb{R} \rightarrow D$ be sufficiently many times differentiable such that

$$
g^{(j)}\left(x^{*}\right)=0, \quad 2 \leq j \leq m-1 \quad \text { and } \quad g^{(m)}\left(x^{*}\right) \neq 0,
$$

for some integer $m,(m \geq 2)$.

In order to show that $h$ given by Equation $(2-5)$ can be combined with $g$, one needs to show that the fixed point $x^{*}$ is hyperbolic for $h$.

Lemma 2.6. Let $x^{*}$ be a nonhyperbolic fixed point of $g$ such that $g^{\prime}\left(x^{*}\right)=1$, with $g$ satisfying (2-6) and $h$ as in (2-5). Then $h$ is differentiable at $x^{*}$ and $x^{*}$ is an attracting fixed point of $h$ with

$$
0<h^{\prime}\left(x^{*}\right)=1-\frac{1}{m}<1 .
$$

The proof of the above lemma follows by using the Maclaurin series for $g$ and $g^{\prime}$ in the computation (by definition) of $h^{\prime}\left(x^{*}\right)$ (see for instance [Traub 64, Isaacson and Keller 66, Kress 98]).

We have then proved the following theorem.

Theorem 2.7. Let $x^{*}$ be an isolated fixed point of $g: D \subset$ $\mathbb{R} \rightarrow D$ where $g$ is at least $m$ times differentiable in $D$ such that

$$
g^{(j)}\left(x^{*}\right)=0, \quad 2 \leq j \leq m-1 \quad \text { and } \quad g^{(m)}\left(x^{*}\right) \neq 0 .
$$

For the continuous extension $h$ of $C(x, g)$ given in (2-5), the iteration function $H=C(g, h)$ is flat.

\section{APPLICATIONS}

Any suitable choice of $h$ can be used in order to obtain a flat iterator $H=C(g, h)$. Since Newton's iteration function, $N(x)$, is the most popular method in the applications it is natural to choose $N$ as the companion $h$ to a given nonhyperbolic iterator $g$. Of course, there are many other alternatives; namely several operators (with appropriate modifications) available from the convergence acceleration area.

In Section 3.1, we begin by showing that Newton's method can be viewed as a particular combined IF. When properly applied, the results of Section 2 allow us to recover well-known properties of Newton's method. We end this section with several numerical examples which illustrate some properties of the combined iterators.

\subsection{Combined Iteration Functions and Newton's Method}

As it is well known, Newton's iteration function $N(x)=$ $x-f(x) / f^{\prime}(x)$ is not well-defined at multiple roots of the equation $f(x)=0$, or equivalently at the $\mathrm{NH}$ fixed points of $g(x)=x-f(x)$. This means that the corresponding sequence of iterates for $N$ is either slowly convergent to the fixed point or not convergent at all. The combined iteration functions $N_{H}=C(g, N)$ and $N_{C}=C(x, N)$ deal with both hyperbolic and nonhyperbolic fixed points. Under mild assumptions, the iterative processes associated to $N_{H}$ and $N_{C}$ will converge faster to a fixed point (if one starts sufficiently close to it) than the one associated to $N$ since $N_{C}$ and $N_{H}$ are flat IF. Hereafter when we say, for instance, that $N_{C}(x)$ converges to $x^{*}$, we mean that the associated iterative process $x_{n}=N_{C}\left(x_{n-1}\right)$ will converge to $x^{*}$.

Consider the equation $f(x)=0$ where $f$ will be assumed to be sufficiently differentiable in a suitable domain. A root $x^{*}$ of $f(x)=0$ is a fixed point of the iteration function $g(x)=x-f(x)$. Newton's iteration function is a particular case of the combined iteration function $(2-2)$ if $x^{*}$ is a simple root $\left(f^{\prime}\left(x^{*}\right) \neq 0\right)$ :

$$
\begin{aligned}
C(x, g) & =\frac{x-f(x)-x\left(1-f^{\prime}(x)\right)}{f^{\prime}(x)} \\
& =\frac{x f^{\prime}(x)-f(x)}{f^{\prime}(x)}=N(x) .
\end{aligned}
$$

The notion of order of convergence of an iterative process (see [Traub 64]) can be rephrased in terms of the classification of a fixed point as follows: When $x^{*}$ is an attracting or super-attracting fixed point of an iteration function $g$, the iterative process $x_{n+1}=g\left(x_{n}\right)$ has order of convergence 1 or at least 2 .

The next proposition states some classical results on Newton's method (see [Ostrowski 73, Isaacson and Keller 66, Holmgren 96, Kress 98]), recovered here as straightforward applications of the results obtained for combined iteration functions. We always assume that $N$ is convergent to $x^{*}$.

Proposition 3.1. Let $x^{*}$ be a root of $f(x)=0$ where $f$ is at least $m$ times continuously differentiable in a neighborhood of $x^{*}$.

(i) If $x^{*}$ is a simple root, then Newton's method has order of convergence at least 2.

(ii) If $x^{*}$ is a root of multiplicity $m(m \geq 2)$, then Newton's method has convergence of order 1 and the 


\begin{tabular}{|c|c|}
\hline Fixed point $x^{*}$ & Flat iteration function \\
\hline$g^{\prime}\left(x^{*}\right) \mid \neq 1$ & $C(x, g)=\frac{g-x g^{\prime}}{1-g^{\prime}} \quad$ (Cor. 2.3) \\
\hline$g^{\prime}\left(x^{*}\right)=1$ and $h^{\prime}\left(x^{*}\right) \neq 1$ & $C(g, h)=\frac{h-g h^{\prime}}{1-h^{\prime}} \quad$ (Thm. 2.5) \\
\hline$g^{\prime}\left(x^{*}\right)=1$ or $h^{\prime}\left(x^{*}\right)=0$ & $C(g, h)=\frac{h-g h^{\prime}}{1-h^{\prime}} \quad$ (Prop. 2.2) \\
\hline$g^{\prime}\left(x^{*}\right)=-1$ & $C(x, g)=\frac{g-x g^{\prime}}{1-g^{\prime}} \quad($ Thm. 2.7) \\
\hline$g^{\prime}\left(x^{*}\right)=-1$ and $h^{\prime}\left(x^{*}\right) \neq-1$ & $C(g, h) \stackrel{\text { def }}{=} \frac{h+g h^{\prime}}{1+h^{\prime}}$ \\
\hline$x^{*}$ simple root for $f(x)=0$ & $C(x, x-f)=x-\frac{f}{f^{\prime}}$ \\
\hline$x^{*}$ simple or multiple root of & $\begin{array}{c}(\text { Prop. 3.1 }(i)) \\
f(x)=0\end{array}$ \\
or $\quad C(x, N)=\frac{N-x N^{\prime}}{1-N^{\prime}}$ \\
$($ Thm. 3.2) \\
$N_{H}=C(g, N)=\frac{N-g N^{\prime}}{1-N^{\prime}}$ \\
\hline
\end{tabular}

TABLE 1. Flat iteration functions for specified fixed points.

respective asymptotic convergence factor is

$$
0<N^{\prime}\left(x^{*}\right)=1-\frac{1}{m}<1 \text {. }
$$

Proof:

(i) A simple root $x^{*}$ of $f(x)=0\left(f^{\prime}\left(x^{*}\right) \neq 0\right)$ is a hyperbolic fixed point of $g(x)=x-f(x)$. So, by Corollary $2.3, x^{*}$ is a super attractor for $N(x)=C(x, g(x))$.

(ii) A root $x^{*}$ of multiplicity $m$ of $f(x)=0$ satisfies $f\left(x^{*}\right)=f^{\prime}\left(x^{*}\right)=\ldots=f^{(m-1)}\left(x^{*}\right)=0$ and $f^{(m)} \neq 0$ (see [Henrici 64], Chap.2). So, the functions $g(x)=x-f(x)$ and the continuous extension $h$ of $N(x)=C(x, g)$ given by $(2-5)$ satisfy the hypotheses of Lemma 2.6, so $N^{\prime}\left(x^{*}\right)=1-1 / m$.

Let us now construct the following iteration functions based on $N$ :

$$
\begin{aligned}
& N_{C}=C(x, N)=\frac{N(x)-x N^{\prime}(x)}{1-N^{\prime}(x)}, \\
& N_{H}=C(g, N)=\frac{N(x)-g(x) N^{\prime}(x)}{1-N^{\prime}(x)} .
\end{aligned}
$$

It is easy to conclude that $N_{C}$ is just Newton's iteration function applied to $\mu(x)=f(x) / f^{\prime}(x)$, a well-known modification of Newton's method (see [Burden 89]) used for the computation of multiple roots of the equation $f(x)=0$.

At a (isolated) root $x^{*}$, either simple or multiple, of $f(x)=0$ (and $g(x)=x-f(x))$ both $N_{C}$ and $N_{H}$ are well-defined at $x^{*}$ since $N^{\prime}\left(x^{*}\right) \neq 1$. From our results on flat iteration functions, namely Theorem 2.5, both $N_{C}$ and $N_{H}$ are flat, that is, the corresponding iterative processes for $N_{C}$ and $N_{H}$ have convergence of order at least 2 .

Theorem 3.2. If $x^{*}$ is either a simple or multiple root of $f(x)=0$ and both $N_{C}(x), N_{H}(x)$ converge to $x^{*}$, then the corresponding iterative processes have convergence of order at least 2.

Theorem 2.5 also gives conditions for a flat iterative process to have convergence of order at least 3 , that is, when the respective flat iteration function is super flat (see Example 3.5(b)). Although $N_{C}$ and $N_{H}$ are both flat, one of them can perform better than the other on the computation of a nonhyperbolic fixed point of the initial function. For instance, in Example 3.3 (see Section 3.2), $N_{C}$ gives exactly the fixed point in only one iteration while $N_{H}$ is only flat.

Table 1 summarizes some flat iterators to choose depending on the assumptions made about the respective fixed point to be computed.

\subsection{NUMERICAL EXPERIMENTS}

The examples were chosen to illustrate the behavior of several combined iteration functions for nonhyperbolic fixed points of the input function $g$, namely $H=C(g, h)$, $C(x, g), N_{H}=C(g, N)$ and $N_{C}=C(x, N)$. The results of the previous sections predict that a flat IF will allow us to pass from a slow iterative process to a fast one. For instance, in Example 3.3, utilizing only three iterations we compute a nonhyperbolic fixed point for certain iteration functions studied in [Sablonnière 87, Sablonnière 91] obtaining a very high precision approximation of the 
fixed point with our combined iteration function $N_{H}$ or even such fixed point in one iteration using $N_{C}$.

Example 3.4 shows a flat iteration function $H=$ $C(g, h)$ for a given IF, $g$, with a nonhyperbolic fixed point. In Example 3.5, an initial slow iterative process having first order of convergence leads to a process of order 3 obtained by means of $N_{H}$.

Example 3.6(a) shows the appearance of extraneous fixed points at points where Newton's IF is not defined. This is just the natural consequence of $N_{C}$ being always well-defined in these points. Moreover, as can be seen in Example 3.6(b), extraneous fixed points can also appear at points where the initial IF is well-defined.

In Examples 3.4-3.6, we graph both the initial IF and a combined one. Graphing the IF allows one, by simple inspection, to get a quick overview of the dynamics involved.

Tables for the first iterates are also given illustrating the numerical behavior of the initial iterator and of the corresponding combined one. In all examples, only a few iterates are sufficient to obtain the fixed points with high accuracy, although the number of iterations should not, in general, be increased too much since numerical instability can occur.

The following numerical examples have been obtained using Mathematica [Wolfram 96]. Computations were carried out with 40 digits of precision.

Example 3.3. Fixed point sequences generated by a process $x_{n+1}=g\left(x_{n}\right)$ converging to a nonhyperbolic fixed point $x^{*}$ are also designated as logarithmic sequences in the sense that $\lim _{n \rightarrow \infty} e_{n+1} / e_{n}=1$, where $e_{n}=x^{*}-x_{n}$. The set of logarithmic sequences whose errors have an asymptotic expansion of the form

$$
e_{n+1}=e_{n}+\sum_{k \geq 1}^{\infty} \alpha_{k} e_{n}^{1+k r}, \alpha_{1}<0 \text { and integer } r \geq 1
$$

has been extensively used as a standard for assessing the quality of several sequence-to-sequence transformations used to accelerate the convergence of such a process.

In [Sablonnière 87, Sablonnière 91] and subsequent papers, the author studied the logarithmic sequences obtained by iterating

$$
g(x)=x-x^{r}, \quad 1 \leq r \leq 6, \quad x_{0}=0.5 \quad \text { where } x^{*}=0
$$

The best accelerator found by Sablonnière shows a precision of about $10^{-10}$ after 19 iterations, the higher precision being obtained for the lower values of $r$.

\begin{tabular}{|c|l|l|}
\hline$r=1$ & \multicolumn{1}{|c|}{$r=10$} & \multicolumn{1}{c|}{$r=20$} \\
\hline 0.5 & 0.5 & 0.5 \\
0 & 0.0087891 & 0.0000181198 \\
0 & $2.475510^{-20}$ & $2.765810^{-94}$ \\
0 & $7.779010^{-196}$ & $1.304110^{-1870}$ \\
\hline
\end{tabular}

TABLE 2. First three iterations of $N_{H}=C(g, N)$, where $g(x)=x-x^{r}$.

Compared to the results of Sablonnière, it is quite impressive to see that our flat iteration $N_{H}$, applied to $g$ in (3-1) for $1 \leq r \leq 20$, achieves a much higher precision after only three iterations. The reason for our good numerical results is that $N_{H}$ is a superflat iteration function for this kind of sequence;

$$
N(x)=\frac{r-1}{r} x, \quad N^{\prime}(x)=1-\frac{1}{r},
$$

so

$$
N_{H}(x)=\frac{N(x)-g(x) N^{\prime}(x)}{1-N^{\prime}(x)}=(r-1) x^{r} .
$$

Thus for $x_{n+1}=N_{H}\left(x_{n}\right)=(r-1) x_{n}^{r}$, we get $\lim _{n \rightarrow \infty} \frac{x_{n+1}}{x_{n}^{r}}=r-1$, so this sequence has order of convergence $r$ for $r>1$ and $x^{*}=0$ is obtained in only one iteration for $r=1$. Table 2 shows the first three iterates for $N_{H}=C(g, N)$ where $g(x)=x-x^{r}$ and $r=1,10,20$.

For this example, $N_{C}$ is optimal in the sense that the fixed point is obtained exactly in one iteration:

$$
N_{C}=C(x, N)=\frac{N-x N^{\prime}}{1-N^{\prime}}=(r-1) x-x(r-1)=0 .
$$

Example 3.4. (See Figure 1 and Table 3.) In this example, the initial IF $g$ is compared with $H=C(g, h)$, where $h=C(x, g)$. In part (a), $x^{*}$ is a nonhyperbolic fixed point with $g^{\prime}\left(x^{*}\right)=1$ whereas in $(\mathrm{b}), g^{\prime}\left(x^{*}\right)=-1$.

(a) Let $g(x)=1+\ln (x)$. This function has the $\mathrm{NH}$ fixed point $x^{*}=1$ with $g^{\prime}(1)=1$. Let $h$ be the continuous extension of $C(x, g)$ :

$$
h(x)=\frac{x \ln (x)}{x-1}, \quad \text { if } \quad x \neq 1 \quad \text { and } \quad h(1)=1 .
$$

The iteration function $H=C(g, h)$ is

$$
\begin{gathered}
H(x)=\frac{1-x+\left(2-2 x+x^{2}\right) \ln (x)+\ln ^{2}(x)}{2-3 x+x^{2}+\ln (x)} \\
\text { for } x \neq 1 \text { and } H(1)=1 .
\end{gathered}
$$

By Theorem 2.5, the iteration function $H$ is flat since $h^{\prime}(1)=1 / 2 \neq 1$ (by Lemma 2.6).

(b) Let $g(x)=x-\ln \left(x^{2}\right)$. This IF has the NH fixed point $x^{*}=1$ with $g^{\prime}(1)=-1$. Let $h$ be $C(x, g)$ :

$$
h(x)=x-\frac{x \ln \left(x^{2}\right)}{2},
$$


(a)

\begin{tabular}{|l|l|}
\hline \multicolumn{1}{|c|}{$\left(x_{n}\right)$} & \multicolumn{1}{c|}{$\left(y_{n}\right)$} \\
\hline 1.5 & 1.5 \\
1.4054651081081643820 & 1.10142617280551354 \\
1.3403682858041913949 & 1.00717661432426 \\
1.2929444163535657425 & 1.00004235 \\
\hline
\end{tabular}

(b)

\begin{tabular}{|l|l|}
\hline \multicolumn{1}{|c|}{$\left(x_{n}\right)$} & \multicolumn{1}{c|}{$\left(y_{n}\right)$} \\
\hline 0.9 & 0.9 \\
1.1107210313156525753 & 0.9811754777692121664 \\
0.9007022669120995963 & 0.9994466194247491503 \\
1.1098633136359449272 & 0.9999995401177632299 \\
0.9013895798894140186 & 0.9999999999996827622 \\
1.1090250373113361886 & 1.0000000000000000000 \\
\hline
\end{tabular}

TABLE 3. (a) $x_{0}=y_{0}=1.5, x_{n}=1+\ln \left(x_{n-1}\right)$, $y_{n}=H\left(y_{n-1}\right), \quad n=1: 3 ; x^{*}=1$ (see Figure 1(a)); (b) $x_{0}=y_{0}=0.9, x_{n}=x_{n-1}-\ln \left(x_{n-1}^{2}\right), y_{n}=H\left(y_{n-1}\right)$, $n=1: 5$ (see Figure 1(b)).

and $H=C(g, h)$,

$$
H(x)=\frac{2 x-\ln ^{2}\left(x^{2}\right)}{2+\ln \left(x^{2}\right)} \quad \text { for } \quad x \neq 0 .
$$

Both $h$ and $H$ are flat since $h^{\prime}(1)=H^{\prime}(1)=0$.

For the same initial point $x_{0}=y_{0}=1.5$, the left-hand side of Table 3 (a) shows the first 3 iterates for $x_{n}=$ $g\left(x_{n-1}\right)$ when $g(x)=1+\ln (x)$, and the right-hand side displays the corresponding iterates for $y_{n}=H\left(y_{n-1}\right)$. In the left-hand side of Table $3(\mathrm{~b})$, the first 5 iterations of $x_{n}=g\left(x_{n-1}\right)$ when $g(x)=x-\ln \left(x^{2}\right)$ are given, and the right-hand side displays the corresponding iterates $y_{n}=H\left(y_{n-1}\right)$. In this case, the initial points are $x_{0}=$ $y_{0}=0.9$.

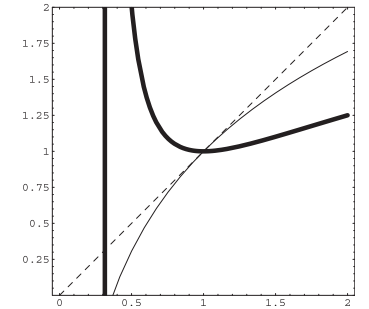

(a)

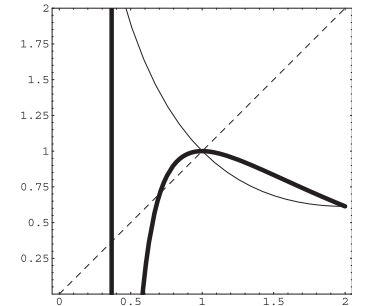

(b)
FIGURE 1. In the figure, $g$ is plotted thinner than $H=$ $C(g, h)$. The fixed point is $x^{*}=1$ : (a) $g(x)=1+$ $\ln (x), g^{\prime}\left(x^{*}\right)=1 ; \quad(\mathrm{b}) \quad g(x)=x-\ln \left(x^{2}\right), g^{\prime}\left(x^{*}\right)=-1$.

Example 3.5. (See Figure 2 and Table 4.) In this example, we compare $N$ with $N_{H}$ for the iteration function $g(x)=\sin (x)$. In [Brezinski and Redivo Zaglia 91, page 325], there are numerical results for nine sequenceto-sequence transformations for the computation of the fixed point $x^{*}=0$ of $g$. The number of exact digits obtained by the application of the referred sequence trans- (a)

\begin{tabular}{|l|l|}
\hline \multicolumn{1}{|c|}{$\left(x_{n}\right)$} & \multicolumn{1}{|c|}{$\left(y_{n}\right)$} \\
\hline 1.0 & 1.0 \\
0.8414709848078965067 & 0.6551450720424305085 \\
0.7456241416655578889 & 0.4335903683634929531 \\
0.6784304773607402290 & 0.28814840089250120018 \\
0.6275718320491591389 & 0.19183231215063892737 \\
\hline
\end{tabular}

(b)

\begin{tabular}{|l|l|}
\hline \multicolumn{1}{|c|}{$\left(x_{n}\right)$} & \multicolumn{1}{|c|}{$\left(y_{n}\right)$} \\
\hline 1.0 & 1.0 \\
0.6551450720424305085 & 0.3361766585172519082 \\
0.4335903683634929531 & 0.014905130258776021077 \\
0.28814840089250120018 & $1.324496388499297754610^{-6}$ \\
0.19183231215063892737 & $0.10^{-17}$ \\
\hline
\end{tabular}

TABLE 4. $g(x)=\sin (x)$ and $x^{*}=0$ : (a) $x_{0}=y_{0}=1$, $x_{n}=g\left(x_{n-1}\right), y_{n}=h\left(y_{n-1}\right), n=1: 4 ;$ (b) $x_{0}=y_{0}=$ $1, x_{n}=h\left(x_{n-1}\right), y_{n}=H\left(y_{n-1}\right) n=1: 4$.

formations, using 15 iterations and $x_{0}=0.5$ as starting point, ranges from 0.82 to 6.20 for the best one. In Table 4 (b) we show that only 4 iterations of $N_{H}$ are necessary for obtaining 17 exact digits.

(a) $g(x)=\sin (x)$ for which $x^{*}=0$ is a nonhyperbolic fixed point. We compare $g$ with the continuous extension of $C(x, g)$ :

$$
\begin{aligned}
C(x, g)=h(x)= & \frac{x \cos (x)-\sin (x)}{\cos (x)-1}, \\
& \text { if } x \neq 0, \quad \text { and } \quad h(0)=0 .
\end{aligned}
$$

As $g^{(2)}(0)=0$ and $g^{(3)}(0)=-1 \neq 0$, then by Lemma 2.6, $x^{*}=0$ is an attracting fixed point for $h$. The corresponding iterative process for $h$ has asymptotic convergence factor $h^{\prime}(0)=2 / 3$.

(b) Now compare the function $h$ obtained in (a) with $H=C(g, h)$. We know from Theorem 2.5 that $H$ is flat. In fact, $H$ is super-flat since $H^{(2)}(0)=0$. Since $H^{(3)}(0)=12 / 5$, the iterative process associated to $H$ has convergence of order 3 .

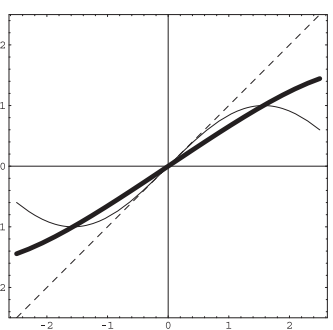

(a)

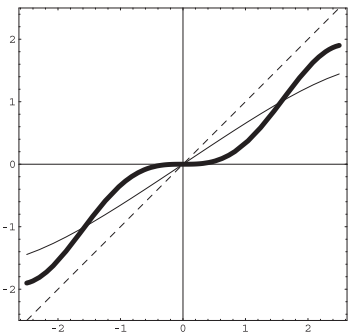

(b)
FIGURE 2. $g(x)=\sin (x)$. (a) The plot of $g$ is thinner than the plot of $h=C(x, g)$. The fixed point $x^{*}=0$ is nonhyperbolic for $g$; (b) Plots for $h$ (thinner) and the flat IF $H=C(g, h)$. 
Example 3.6. (See Figure 3 and Tables 5 and 6.) In this example, we compare in (a) the iteration function $N$ and $N_{C}=C(x, N)$, and in (b) the iteration functions $N$ and $N_{H}=C(g, N)$. The test function for both cases (a) and (b) is the polynomial

$$
p(x)=-3(x-2)^{4}(x-1)^{3} x^{5},
$$

which has three roots $x^{*}=0,1,2$ of multiplicities 5,3 , and 4 , respectively.

(a) We compare the behavior of the iteration function $N(x)=x-p(x) / p^{\prime}(x)$ and $N_{C}(x)=C(x, N(x))$ having the following expression

$$
N_{C}(x)=\frac{x^{2}\left(20-28 x+11 x^{2}\right)}{20-60 x+81 x^{2}-50 x^{3}+12 x^{4}} .
$$

Table 5 shows the values of the derivatives of $N$ and $N_{C}$ at the respective fixed points. This table confirms that Newton's process has convergence of order 1 , while the iterative process associated to $N_{C}$ is of order 2. Extraneous fixed points of $N_{C}$ occur at points of discontinuity of $N$.

(b) We compare $N(x)$ and $N_{H}(x)=C(g(x), N(x))$ where $g(x)=x-p(x)$. In this case, we will not display the expression of $N_{H}(x)$ since it is too long. The iterative process associated to $N_{H}$ is, as in (a), of order 2 (for any of the roots of $p(x)=0$ ). As one can see from Figure $3(\mathrm{~b}), N_{H}$ has extraneous fixed points.

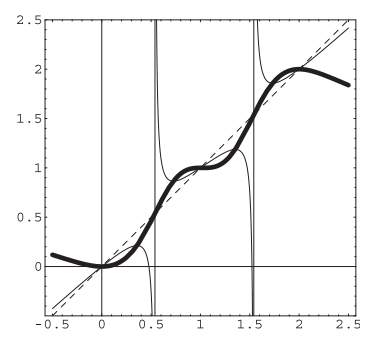

(a)

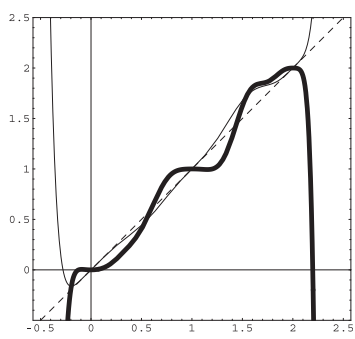

(b)
FIGURE 3. $p(x)=-3(x-2)^{4}(x-1)^{3} x^{5}$ : (a) $N$ thinner than $N_{C}=C(x, N)$; (b) $g(x)=x-p(x)$ thinner than $N_{H}$.

\begin{tabular}{|c|c|c|c|}
\hline$x^{*}$ & $N^{\prime}\left(x^{*}\right)$ & $N_{C}^{\prime}\left(x^{*}\right)$ & $N_{C}^{(2)}\left(x^{*}\right)$ \\
\hline 0 & $4 / 5$ & 0 & 2 \\
1 & $2 / 3$ & 0 & $-2 / 3$ \\
2 & $3 / 4$ & 0 & $-11 / 4$ \\
\hline
\end{tabular}

TABLE 5. Derivatives for $N$ and $N_{C}$ for Example 3.6(a). (a)

\begin{tabular}{|l|l|}
\hline \multicolumn{1}{|c|}{$\left(x_{n}\right)$} & \multicolumn{1}{c|}{$\left(y_{n}\right)$} \\
\hline 0.2 & 0.2 \\
0.14744525547445256183 & 0.05466332694857817110 \\
0.11202555417067730826 & 0.003260926997125556906 \\
0.08647332273470264775 & 0.000010689272708733296725 \\
0.06740643461577624390 & $1.142625052381464703210^{-10}$ \\
\hline
\end{tabular}

\begin{tabular}{|l|l|}
\hline \multicolumn{1}{|c|}{$\left(x_{n}\right)$} & \multicolumn{1}{c|}{$\left(y_{n}\right)$} \\
\hline 0.2 & 0.2 \\
0.19484021964800001001 & 0.06377257819031443284 \\
0.19017122775166904930 & 0.004624878730215735896 \\
0.18591940778245240134 & 0.000021548768367953577176 \\
0.18202560991039300755 & $4.64365428309801254710^{-10}$ \\
\hline
\end{tabular}

TABLE 6. $p(x)=-3(x-2)^{4}(x-1)^{3} x^{5}$ and $x^{*}=0: \quad$ (a) $x_{0}=0.2, x_{n}=N\left(x_{n-1}\right), y_{n}=N_{C}\left(y_{n-1}\right), n=1: 4$; (b) $x_{0}=0.2, x_{n}=x_{n-1}-p\left(x_{n-1}\right), y_{n}=N_{H}\left(y_{n-1}\right)$, $n=1: 4$.

\section{Acknowledgments}

The author thanks the anonymous referees for suggesting a number of improvements. This work was supported by Instituto de Mecânica-IDMEC/IST, Centro de Projecto Mecânico, through FCT (Portugal)/program POCTI.

\section{REFERENCES}

[Brezinski and Redivo Zaglia 91] C. Brezinski and M. Redivo Zaglia. Extrapolation Methods Theory and Practice. Amsterdam: North-Holland, 1991.

[Burden 89] R. L. Burden and J. D. Faires. Numerical Analysis, Fourth edition. Boston: PWS-KENT, 1989.

[Epureanu and Greenside 98] B. I. Epureanu and H. S. Greenside. "Fractal Basins of Attraction Associated with a Damped Newton's Method." SIAM Rev. 40 (1998), 102-109.

[Gerlach 94] J. Gerlach. "Accelerated Convergence in Newton's Method." SIAM Rev. 36 (1994), 272-276.

[Gilbert 94] W. J. Gilbert. "Newton's Method for Multiple Roots." Computers and Graphics 18 (1994), 227-229.

[Golubitsky and Schaeffer 85] M. Golubitsky and D. G. Schaeffer. Singularities and Groups in Bifurcation Theory, Vol. I. New York: Springer-Verlag, 1985.

[Govaerts 00] W. Govaerts. Numerical Methods for Bifurcations of Dynamical Equilibria. Philadelphia: SIAM, 2000 .

[Henrici 64] P. Henrici. Elements of Numerical Analysis. New York: John Wiley and Sons, 1964.

[Holmgren 96] R. A. Holmgren. A First Course in Discrete Dynamical Systems. New York: Springer-Verlag, 1996.

[Isaacson and Keller 66] E. Isaacson and H. B. Keller. Analysis of Numerical Methods. New York: John Wiley and Sons, 1966.

[Kress 98] R. Kress. Numerical Analysis. New York: Springer-Verlag, 1998. 
[Ostrowski 73] A. M. Ostrowski. Solutions of Equations in Euclidean and Banach Spaces. Third edition. New York: Academic Press, 1973.

[Sablonnière 87] P. Sablonnière. "Convergence Acceleration of Logarithmic Fixed Point Sequences." J. Comput. Appl. Math. 19 (1987), 55-60.

[Sablonnière 91] P. Sablonnière. "Comparison of Four Algorithms Accelerating the Convergence of Some Logarithmic Fixed Point Sequences." Numer. Algorithms 1 (1991), 177-198.
[Traub 64] J. F. Traub. Iterative Methods for the Solution of Equations. Englewood, NJ: Prentice-Hall, 1964.

[Vrscay and Gilbert 88] E. R. Vrscay and W. J. Gilbert. "Extraneous Fixed Points, Basin Boundaries and Chaotic Dynamics for Schröder and König Rational Iteration Functions." Numer. Math. 52 (1988), 1-16.

[Wolfram 96] S. Wolfram. The Mathematica Book, Third edition. Cambridge: Cambridge University Press, 1996.

Mário M. Graça, Instituto Superior Técnico, Dep. Matemática, Av. Rovisco Pais, 1049-001 Lisboa, Portugal (mgraca@math.ist.utl.pt)

Received October 20, 2000; accepted in revised form June 30, 2001. 